\title{
IMAGE CHARGE UNDULATOR: THEORETICAL MODEL AND TECHNICAL ISSUES *
}

\author{
Yuhong Zhang, Yaroslav Derbenev, James R. Boyce, Rui Li \\ Jefferson Lab, Newport News, VA 23606, USA
}

\section{Abstract}

A relativistic electron beam undergoes undulating motion due to its image charge wakefields while passing close to a conducting grating surface. A new device, an image charge undulator, has been proposed recently [1] to utilize this mechanism for generating coherent hard radiation. We demonstrate the physics principle of this device by a $2 \mathrm{D}$ model of a uniform sheet beam. The transverse image charge wakefields, synchrotron radiation frequency and coherent radiation gain length are presented. We discuss a proof-of-principle experiment that takes into consideration such technical issues as grating fabrication, flat beams and beam alignment.

\section{INTRODUCTION}

A relativistic electron beam passing near a metal surface generates an electric polarization (image charges) of the surface, which applies a Lorentz force (image charge wakefields) back on the beam. These wakefields become wiggler type fields when the metal surface is a grating. The electron beam undergoes undulating motion due to these wakefields and emits/amplifies radiation just as in a conventional magnetic undulator. To enhance wakefields and also to stabilize the electron beam, we close the single grating by a second identical surface shown in Fig. 1. Such an asymmetric periodic structure is named an image charge undulator (ICU).

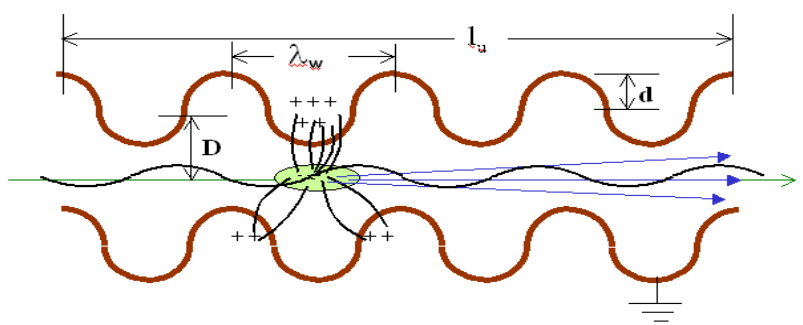

Figure 1. Schematic drawing of image charge undulator.

Let us consider a uniform sheet beam (surface charge density $\sigma_{0}$ ) in an infinitely long planar undulator as shown in Fig. 1. In this case, the alternating component of magnetic field vanishes, while the alternating electric field coincides with electrostatic solution for a charged sheet of the same density. The transverse image charge wakefields (defined as the total field minus the field of the source charges) have the following general form

$$
E_{x}(x, z)=\sum_{n=1}^{\infty} E_{x n}(x) \sin \left(n k_{w} z+\varphi_{x z}\right)
$$

to reflect periodicity in the longitudinal direction, where $\varphi_{\mathrm{xn}}$ are phases, $k_{w}=2 \pi / \lambda_{w}$, and $\lambda_{w}$ is the period of the

* This work was supported by the U.S. Dept. of Energy under contract DE-AC05-84-ER40150. structure. Due to the linearity of Maxwell equations,

$$
E_{x n}(x) \sim E_{0}
$$

for all $n$, where $E_{0}=2 \pi \sigma_{0}$ is the static electric field of a uniform sheet charge in free space. This relation implies that the image charge wakefields are always proportional to the surface charge density of the sheet beam, i.e., total charge and dimensions of a flat bunch.

Normally, higher harmonic terms in Eq. (1) decay very fast so the wakefields are dominated by the first several nonzero terms. Keeping only the first term in Eq. (1), we obtain from the electron equation of motion that

$$
v_{x}(z) \approx \frac{c K}{\gamma} \cos k_{w} z, \quad K=\frac{e E_{x 1}(0)}{m c^{2} k_{w}}
$$

where $\gamma$ is the Lorentz factor, and $E_{x 1}(x) \approx E_{x 1}(0)$ because $x$ is very small for high energy electrons. This result shows that electrons indeed undergo undulating motion and therefore emit/amplify radiation in a way similar to a conventional magnetic undulator. According to the synchrotron radiation theory, the resonance frequency of this planar undulator is

$$
\lambda=\lambda_{w}\left(1+\frac{1}{2} K^{2}\right) / 2 \gamma^{2}
$$

\section{A SIMPLE 2D MODEL}

The 2D model of ICU shown in Fig. 2 can be viewed as a $2 \mathrm{D}$ waveguide (vertical size $2 D$ ) attached by two sets of identical 2D rectangular cavities (width $L$ and depth $d$ ). Cavities are uniformly distributed along the $z$-axis. The period of this ICU is $2 L$. Both waveguide and cavities are made of perfectly conducting material. A uniform sheet beam (of zero thickness) passes through the center of the ICU. In Ref. 1, we have shown analytically the transverse image charge wakefield at the center of the ICU is

$$
E_{x}(0, z)=E_{0} \sum_{n=0}^{\infty} N_{x 2 n+1} \sin (2 n+1) k_{w} z
$$

The dimensionless coefficients $N_{x 2 n+1}$ depend only on the ICU geometry (i.e., ratios $d / L$ and $D / L$ ), not on parameters of the flat beam, and are given as follows

$$
N_{x 2 n+1}=-\frac{\widetilde{a}_{n} \tanh (2 n+1) \pi \frac{d}{L}}{\left(n+\frac{1}{2}\right) \pi \sinh (2 n+1) \pi \frac{D}{L}}
$$

$\widetilde{a}_{n}$ in Eq. (6) can be solved from linear equations

$$
\widetilde{a}_{n}=1+\sum_{m=0}^{\infty}\left[\widetilde{\Gamma}_{n m}-\widetilde{\Lambda}_{n m}\right] \widetilde{a}_{m}
$$

where

$$
\widetilde{\Gamma}_{n m}=-\frac{2 n+1}{2 m+1} \tanh (2 m+1) \pi \frac{d}{L} \int d \rho \frac{\widetilde{I}_{s n}(\rho) \widetilde{I}_{c n}(\rho)}{i \tanh 2 \pi \frac{D}{L}} \sum_{\alpha} e^{i 2 \alpha \pi}
$$




$$
\widetilde{\Lambda}_{n m}=-\frac{2 n+1}{2 m+1} \tanh (2 m+1) \pi \frac{d}{L} \int d \rho \frac{\widetilde{I}_{s n}(\rho) \widetilde{I}_{c n}(\rho)}{i \sinh 2 \pi \frac{D}{L}} \sum_{\alpha} e^{i(2 \alpha+1) \pi}
$$

are elements of coupling matrices between two eigen modes of cavities on the same or opposite sides of the waveguide, and

$$
\widetilde{I}_{c n}(\rho)=\frac{1}{\pi} \frac{i \rho\left[1+e^{-i \rho}\right]}{(2 n+1)^{2}-\rho^{2}}, \quad \widetilde{I}_{s n}(\rho)=\frac{1}{\pi} \frac{\left[1+e^{+i \rho}\right]}{(2 n+1)^{2}-\rho^{2}}
$$

Further, one can use the following relation

$$
\sum_{\alpha=-N}^{N} e^{i 2 \alpha \pi \rho}=\frac{\sin N \pi \rho}{\sin \pi \rho} \underset{N \rightarrow+\infty}{\longrightarrow} \sum_{l=-\infty}^{+\infty} \delta(\rho-l)
$$

to simplify Eq. (8) and (9).

It should be noted that we have used here a slightly simplified notation from Ref. 1, and have used a tilde on all new variables to denote this difference.

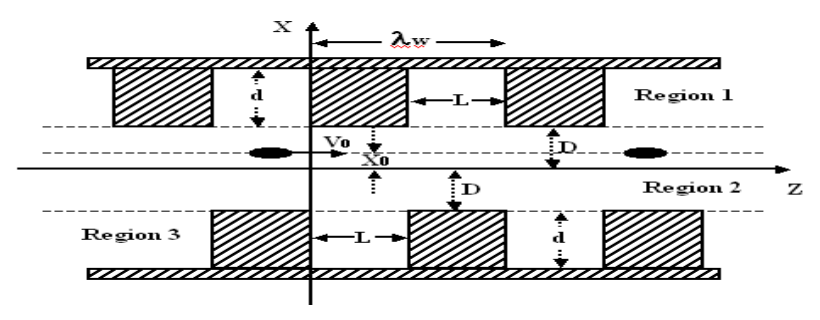

Figure 2. 2D Image charge undulator made of $2 \mathrm{D}$ waveguide and $2 \mathrm{D}$ rectangular cavity.

\section{ASYMPTOTIC BEHAVIOR}

Calculating matrix elements $\widetilde{\Gamma}_{n m}$ and $\widetilde{\Lambda}_{n m}$, and then solving linear equations (7) to find $\widetilde{a}_{n}$ normally require numerical computations. However, it is useful for gaining physics insight to develop an asymptotic formula for $N_{x 2 n+1}$. Considering the fact that $\widetilde{a}_{n}$ are slowly varying numbers in order of one, then

$$
N_{x 2 n+1} \sim-\frac{\tanh (2 n+1) \pi \frac{d}{L}}{\left(n+\frac{1}{2}\right) \pi \sinh (2 n+1) \pi \frac{D}{L}}
$$

In particular, for the first nonzero term, $n=0$,

$$
N_{x 1} \sim-\frac{2}{\pi} \tanh \pi \frac{d}{L} / \sinh \pi \frac{D}{L}
$$

This leads to

$$
\begin{aligned}
& N_{x 1} \rightarrow-2 \frac{d}{L} / \sinh \pi \frac{D}{L} \rightarrow 0, \quad \text { when } \quad d \ll L \\
& N_{x 1} \rightarrow-\frac{2}{\pi} / \sinh \pi \frac{D}{L}, \quad \text { when } \quad d \gg L
\end{aligned}
$$

and

$$
\begin{aligned}
& N_{x 1} \sim-\frac{2}{\pi^{2}} \frac{L}{D} \tanh \pi \frac{d}{L} \rightarrow-\infty, \text { when } \quad D \ll L L \\
& N_{x 1} \sim-\frac{4}{\pi} e^{-\pi \frac{D}{L}} \tanh \pi \frac{d}{L} \rightarrow 0, \text { when } D>L
\end{aligned}
$$

Both Eq. (14) and (17) are well expected since the transverse wakefields vanish when either the cavity depth reduces to zero so the structure becomes symmetric or two gratings are placed too far away. On the other hand, the transverse wakefields reach plateau values as shown in Eq. (15) when the cavity depth is much larger than the undulator period. The wakefields are dominated by the image charges of the nearest metal surface, and since the image charges on the other surface are much farther they can be neglected. Eq. (16) displays a direct inversely proportional relationship between the wakefields and vertical size of the waveguide, i.e., one can increase wakefields by reducing the distance between two gratings.

\section{A NUMERICAL EXAMPLE}

We now present numerical calculations of undulating motion for a flat electron beam of $250 \mathrm{MeV}$ energy in an ICU of $50 \mu \mathrm{m}$ period using the analytical formulas in section 2. The flat bunch is $100 \mu \mathrm{m}$ long, $100 \mu \mathrm{m}$ wide and $4 \mu \mathrm{m}$ thick, and contains $6 \cdot 10^{10}$ electrons (total charge is about $10 \mathrm{nC}$ ). Since the longitudinal bunch size is twice the ICU period, the assumption of infinitely long sheet beam in the $2 \mathrm{D}$ model is only an approximation. We should expect certain deviations from the theoretical model. Other ICU dimensions, i.e., grating tooth depth $d$ and separation of two gratings $2 D$, are parameters of numerical calculations, and their values typically are equal or close to the grating period $\lambda_{\mathrm{w}}$. Matrices $\widetilde{\Gamma}_{n m}$ and $\widetilde{\Lambda}_{n m}$ in Eq. (7) are truncated to 100 by 100 , and we use the Gaussian elimination method to solve Eq. (7).

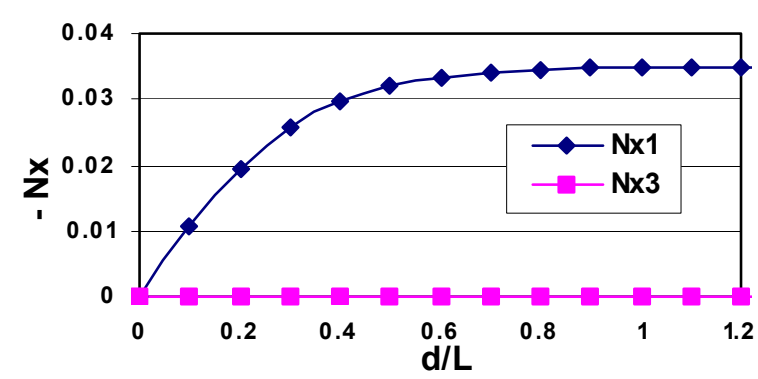

Figure 3. The first two ICU geometry dependent factors as functions of $\mathrm{d} / \mathrm{L}$, when $\mathrm{D} / \mathrm{L}=1$.

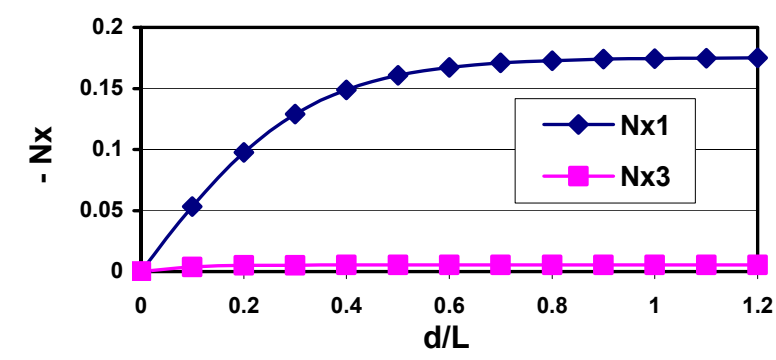

Figure 4. The first two ICU geometry dependent factors as functions of $d / L$, when $D / L=0.5$.

The geometric factors $N_{x 2 n+1}$, as well as the wakefields, are sensitive functions of ratios $D / L$ and $d / L$, as shown in Figs. (3-5). The wakefields quickly reach plateau values when $d$ is larger than $L$, while on the other hand, they can go very high when the two gratings are very close. These behaviors confirm the asymptotical formulas of the previous section. The separation of two gratings is limited by flat beam emittance and alignment, and at the present 
time, $\mathrm{D} \approx 0.5 L \approx 12.5 \mu \mathrm{m}$ is a challenging but still reachable value.

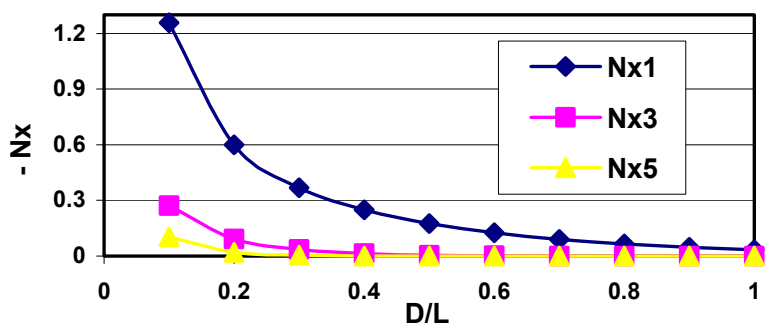

Figure 5. The first three ICU wakefield numerical factors as functions of $\mathrm{D} / \mathrm{L}$, when $\mathrm{d} / \mathrm{L}=1$.

To provide a direct and quantitative comparison between an ICU and a conventional magnetic undulator, we define an equivalent magnetic field derived from the Lorentz force formula

$$
B_{e q v}=E_{x 1}(0) / c
$$

This magnetic field would produce the same strength Lorentz force as the image charge wakefields. It is plotted in Fig. 6 as a function of $D / L$ while $d / L=1$. The values are very significant, and can be as high as $35 \mathrm{~T}$ for $\mathrm{D}=12.5$ $\mu \mathrm{m}$, a value unreachable by conventional undulators or wigglers of current technologies.

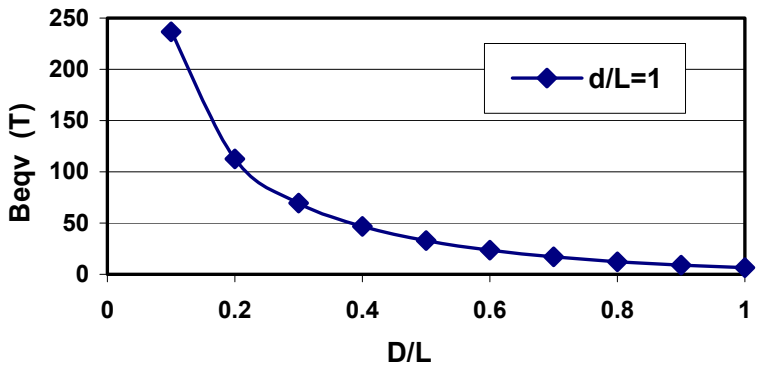

Figure 6. Equivalent magnetic field as a function of $\mathrm{D} / \mathrm{L}$, when $\mathrm{d} / \mathrm{L}=1$

Fig. 7 shows the undulator parameter $K$, ranged from 0.03 to 1.2. The gain length [2] of a FEL process is plotted in Fig. 8. Its range is from 0.3 to $3.4 \mathrm{~cm}$ depending on the separation between two gratings. This points to a very promising large radiation gain and even possible saturation in an ICU of less than $1 \mathrm{~m}$ in length.

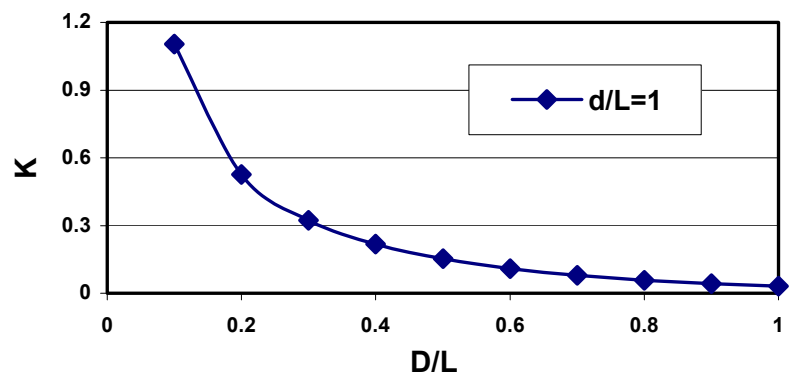

Figure 7. Undulator parameter $\mathrm{K}$ as a function of $\mathrm{D} / \mathrm{L}$, when $\mathrm{d} / \mathrm{L}=1$.

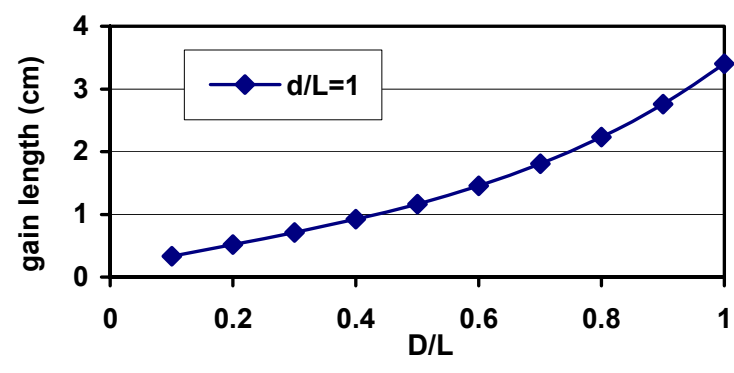

Figure 8. Gain length as a function of $\mathrm{D} / \mathrm{L}$, when $\mathrm{d} / \mathrm{L}=1$.

\section{PROPOSED PROOF-OF-PRINCIPLE EXPERIMENT}

The concept of ICU can be experimentally verified in a straightforward manner. First produce a flat beam with the required characteristics, then send the beam between properly machined and positioned flat plates, and finally monitor the resulting radiation downstream.

Flat beams of a very small x-emittance $(0.01 \mu \mathrm{m}$, norm, or less) can be obtained from electron guns with the cathode immersed in a solenoid field. After acceleration to the energy range of tens of $\mathrm{MeV}$, the electron beam can be ejected from the solenoid and transformed to a flat area, applying the vortex-plane beam adapters [3]. The yemittance then becomes correspondingly large since the geometrical mean of the two emittances is equal to the beam normalized emittance at the cathode. See also [4].

The flat beam is then sent through a beam line cross fitted with standard, remotely controlled, linear motors with alignment guides all in the vacuum. Metallic plates, machined with the desired periodic surface ridges, can be mounted on the motors. (Commercially available machining techniques can cut grating ridges down to as small as $10 \mu \mathrm{m}$.) The plates face each other. Temperature stabilization can be added to back sides of the plates and the e-beam can be pulsed to control induced heating.

Initially, the separation between plates is large so the electron beam can be tuned without striking either plate. When the beam is aligned, the plates are moved towards each other in pre-determined steps. Photon radiation can be monitored downstream as a function of plate separation.

\section{CONCLUSIONS}

A new device, image charge undulator, has been theoretically examined as a potential source for hard radiation with an uncomplicated device. A straightforward experiment is proposed to verify these studies.

\section{REFERENCES}

[1] Y. Zhang, Ya. Derbenev and R. Li, Proceedings of FEL 2003, NIM, in press.

[2] E. L. Saldin, et al., The Physics of Free Electron Lasers, Springer-Verlag (2000)

[3] A. Burov, et al., Phys. Rev. E66: 016503, (2002)

[4] Y. Sun, et al., Proceedings of PAC 2003, (2003) 